Proc. of the 15th Int. Workshop on Slow Positron Beam Techniques and Applications, Prague, September 2-6, 2019

\title{
TOT Method for the Disentanglement of Photons in Positron Annihilation Lifetime Spectroscopy
}

\author{
S.K. Sharma*, on Behalf of the J-PET COllaboration \\ Jagiellonian University, Faculty of Physics, Astronomy and Applied Computer Science, \\ S. Łojasiewicza 11, 30-348, Cracow, Poland
}

\begin{abstract}
The Jagiellonian Positron Emission Tomograph (J-PET) is a multidisciplinary device aiming to perform studies in medical field as well as to test the fundamental symmetries. The plastic scintillators offer very good time resolution. Although, due to the low atomic number of the plastic material, the incident photons interact mainly via the Compton effect. Thus instead of full energy deposition by the photon, there is a range of energy depositions depending on its scattering angle. For the positron annihilation lifetime spectroscopy studies, it is necessary to distinguish the photons emitted from different processes. Therefore, it becomes crucial to find a method to disentangle the photons of different energies. In the present work, the control spectra are discussed which can unambiguously categorize the photons of different energies and origins, which are essential for the studies based on the positronium lifetime.
\end{abstract}

DOI: 10.12693/APhysPolA.137.130

PACS/topics: 24.10.-i, 32.10.-f

\section{Introduction}

Jagiellonian Positron Emission Tomograph is the first tomograph based on the plastic scintillators [1-5]. It is being developed as an alternative for medical imaging which will be several times cheaper than the commercially available tomographs based on crystal scintillators. In the framework of J-PET, to utilize the fast timing feature of the plastic scintillators, time over threshold (TOT) method is adopted as the estimation of energy deposition by photons instead of charge integration method. This method was first proposed by Nygren and Millaud which measures the width of the signal pulse at fix threshold and gives the estimation of signal's amplitude $[6,7]$. Such a conversion between signal width to amplitude is strongly non-linear but can be diminished by applying the multiple thresholds on the obtained signal [8-10]. Furthermore, adopting the TOT approach reduces the readout cost by combining the time and energy information.

J-PET detector possesses the capability to study the lifetime of positronium $(\mathrm{Ps})$ atoms by registering the multiple photons originating from radioactive source and the decays of Ps atoms [11, 12]. The positron annihilation lifetime spectroscopy (PALS) is widely being used in characterizing the nanostructures of materials. J-PET opens the perspectives for PALS applications in medical diagnostics $[13,14]$. In plastic scintillator the photon interacts predominantly via the Compton scattering which hinders the identification of photons of different energies. In order to perform the studies based on positronium lifetime we need to develop the methods to distinguish the photons which can potentially originate from different

*e-mail: sushil.sharma@uj.edu.pl sources e.g., from direct $\mathrm{e}^{+}-\mathrm{e}^{-}$annihilation, decay channels of the positronium atoms (para-positronium (p-Ps) or ortho-positronium (o-Ps) states), and de-excitation photons etc.

In the context of present work, the control spectra measuring eventwise the relative scattering angles between the registered photons is discussed to demonstrate the selection of photons from different origins. Furthermore, TOT spectra which can be efficiently used as a tool to distinguish the photons of different energies will also be elaborated.

\section{Experimental setup}

J-PET is composed of 192 detection modules arranged coaxially in three layers. Each module is composed of a plastic strip (EJ-230) of volume $500 \times 19 \times 7 \mathrm{~mm}^{3}$ connected with R9800 Hamamatsu photomultipliers (PMTs) at each end. The obtained signal is probed at four fixed thresholds $30,160,240$, and $300 \mathrm{mV}$ in voltage domain using a multi-voltage threshold mezzanine (MVT) to attain good approximation for the hit time and hit position of photons within the scintillators [15]. The data is collected in trigger less mode of data stream with rates of 8 Gbps [16]. A small chamber made of polyamide $6(\mathrm{PA} 6)$ of density $1.14 \mathrm{~g} / \mathrm{cm}^{3}$ is placed at the center of the detector geometry. Figure 1 shows the setup picture in the laboratory. A beta emitter ${ }^{22} \mathrm{Na}$ source wrapped in a kapton foil was kept in the center of chamber sandwiched between $\approx 6 \mathrm{~mm}$ layer of XAD4 porous material. The presence of XAD4 enhances the probabilities of the formation of Ps atoms. The material used in making the chamber has high rigidity and mechanical strength. The low density of the chamber material decreases the scattering possibility of high energetic gammas emitting from the source or annihilation photons. 


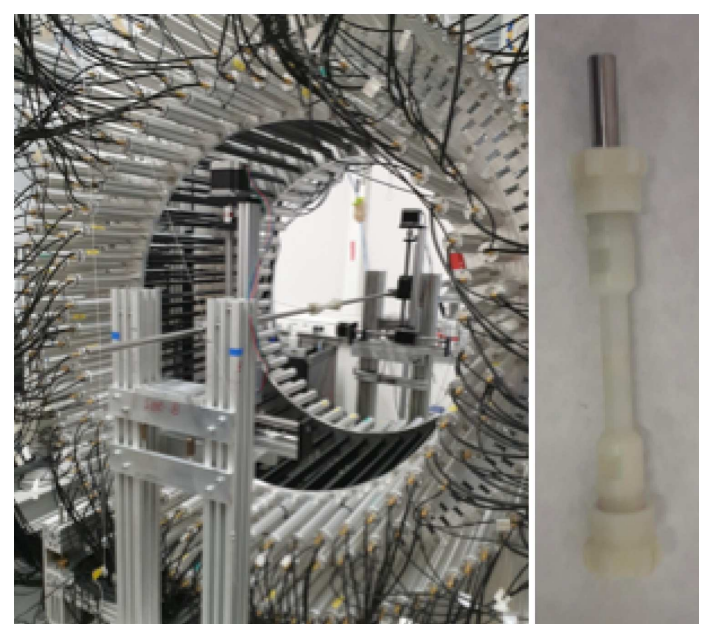

Fig. 1. Photo of the J-PET scanner with small chamber placed at the center. In the adjacent part the closer look of the chamber is shown.

To monitor the experimental condition, a meteo station is used which measures the temperature, humidity, and pressure automatically in the laboratory. For the signal reconstruction, selection, and analysis of collected data, an offline analysis framework is being developed $[17,18]$.

\section{Results}

In the decay of ${ }^{22} \mathrm{Na}$ source photons of two different energies of $511 \mathrm{keV}$ (from $\mathrm{e}^{+}-\mathrm{e}^{-}$annihilation photons) and $1275 \mathrm{keV}$ (prompt photon) are emitted. Furthermore, positron $\left(\mathrm{e}^{+}\right)$from source in interaction with surrounding XAD4 porous material may produce the bound state, a Ps atom which can decay via annihilation in multiple photons varying in energies [0-511 keV] strictly obeying the momentum and energy conservation. Ps atom based on the possible state i.e., p-Ps and o-Ps may decay in even number of photons $(2 n ; n=1,2,3 \ldots)$ or odd number of photons $(2 n+1 ; n=1,2,3, \ldots)$ constrained by the charge conjugation symmetry with maximum probability into two and three, respectively. Thus, the primary photons of different energies can be categorized as follows: (A) prompt photons $(1275 \mathrm{keV})$ plus direct annihilation photons $\left(\mathrm{e}^{+} \mathrm{e}^{-}: 511 \mathrm{keV}\right)$ from source, (B) prompt photons plus the annihilation photons from the decay of $\mathrm{Ps}$ atoms ( $\mathrm{p}$-Ps into two or o-Ps into three with energies in range $0-511 \mathrm{keV})$, and $(\mathrm{C})$ prompt photons plus the annihilation photons from o-Ps to p-Ps conversion i.e., pick-off process. For the analysis, considering the events from all possible cases as mentioned above and to clean the data mainly due to the the multiple scatterings of photons we selected the events with three hits only where hit refers to the photon interaction inside the plastic scintillator. Distribution of relative angles between three hit scintillators ordered from smallest to largest can be used to differentiate the origin of the primary photon.

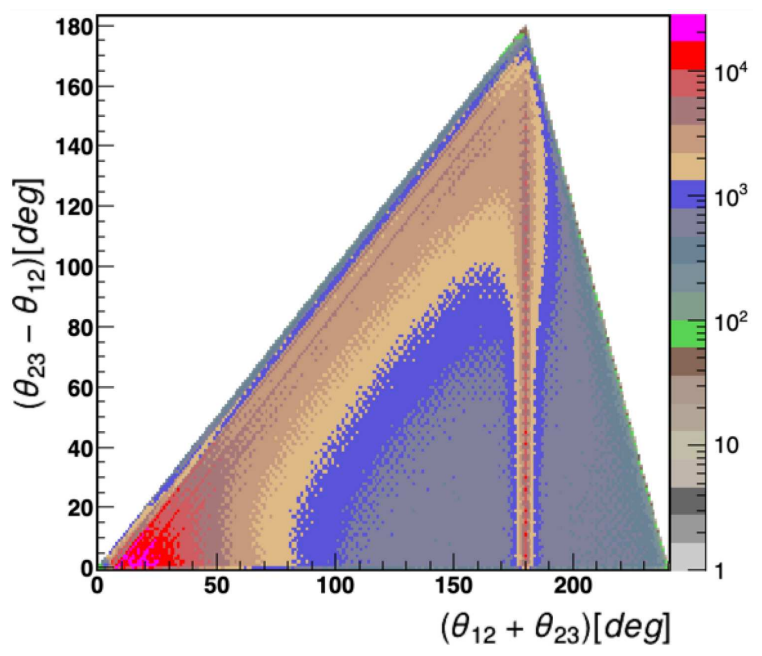

Fig. 2. Distribution of events with 3 hits for sum of two smallest angles $\left(\theta_{12}+\theta_{23}\right)$ versus their difference $\left(\theta_{23}-\theta_{12}\right)$, where each hit refers to the interaction of photon with individual scintillator.

In Fig. 2, the sum of two smallest relative angles between the primary photons is plotted with their difference. Based on the kinematic constraints in order to tag the registered three photons from the decay of o-Ps after arranging the relative angles in ascending order i.e., $\theta_{12} \leq \theta_{23} \leq \theta_{31}$ the region with $\theta_{12}+\theta_{23}>180^{\circ}$ is selected. Similarly, the registered annihilation from the back-to-back photons ordered in time and with single scattering will contribute around $\theta_{12}+\theta_{23} \approx 180^{\circ}$. The region where $\theta_{12}+\theta_{23}<180^{\circ}$ is populated by the events when the one or two hits out of three are from the scatterings of the primary photons [19]. In about $90.34 \%$ decays of ${ }^{22} \mathrm{Na}$ source, a prompt photon of energy $1275 \mathrm{keV}$ is also emitted just after the emission of $\mathrm{e}^{+}$ within the time interval of $\approx 3.7$ ps. However, it is emitted isotropically and shows no angular correlation with the annihilation photons. The emission time of prompt photon is within range of few ps of positron emission, so its registration time can be used as start signal for the formation of Ps atom whereas the registration of annihilation photons will work as Ps decay and hence allow to measure the lifetime of Ps atom.

For an interaction of photon inside the scintillator, the time difference is measured between leading and trailing edges at four fixed thresholds (Fig. 3).

The sum of the time differences for measured signals at all thresholds and at both ends of scintillators gives the total TOT value which is the measure of the total deposited energy by the interacting photon

$$
\mathrm{TOT}=\sum_{\mathrm{PMT}=\mathrm{A}, \mathrm{B}} \sum_{\mathrm{Thr}_{1-4}} \mathrm{TOT}_{\mathrm{PMT}, \mathrm{Thr}} .
$$

The typical TOT spectrum for events with 3 hits without applying any selection criteria on their origins is shown in Fig. 4. 


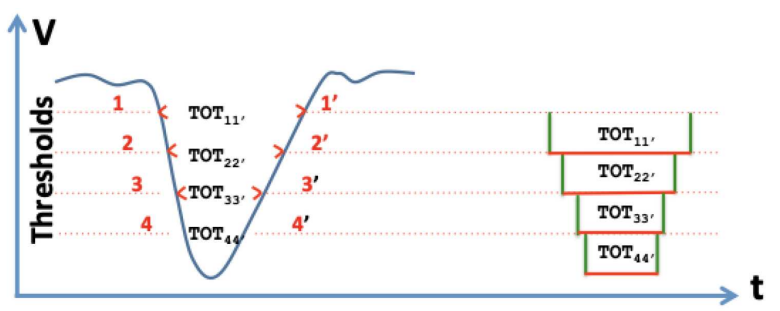

Fig. 3. The pictorial presentation of signal measured and probed at four different thresholds.

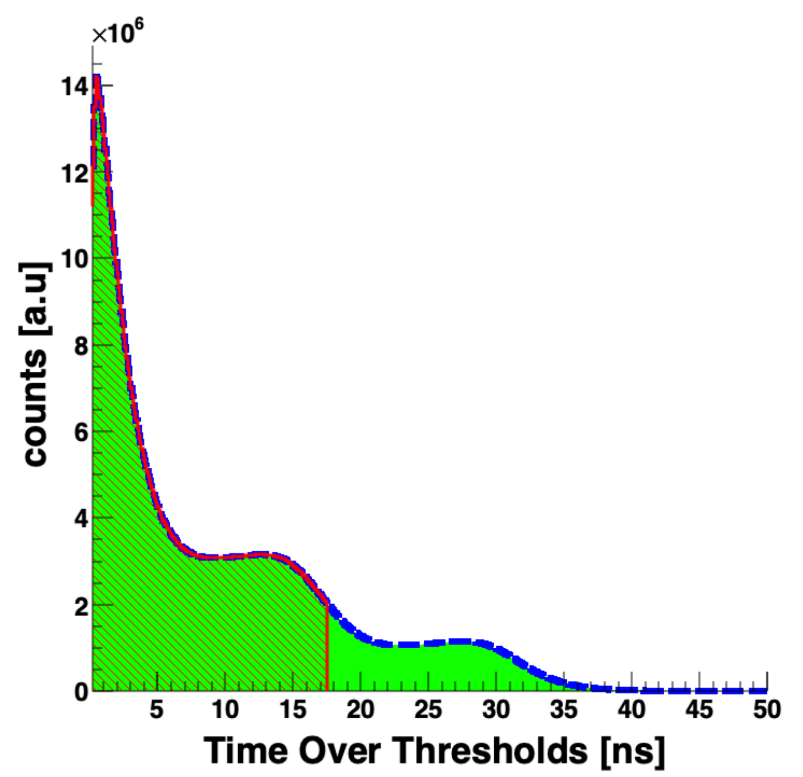

Fig. 4. TOT spectra obtained using the ${ }^{22} \mathrm{Na}$ source sandwiched between XAD4 material placed inside a small chamber made of PA6.

The TOT spectrum resembles the Compton scattering like structure with presence of clear edges. The TOT values are related to the energy deposition in interaction of photon and can be used to select the photons of different incident energies. In Fig. 4, green filled area under the blue dashed line curve has maximum range till $40 \mathrm{~ns}$. It can be conjectured that signals contributing in this region are mainly due to the prompt photon with edge $\approx 32.5$ ns. However, the region up to $17.5 \mathrm{~ns}$ is an overlapping region for TOT values which can have contribution not only from the prompt photon but also from the $\mathrm{e}^{+} \mathrm{e}^{-}$annihilation photons. The TOT value at $17.5 \mathrm{~ns}$ (shown by solid red line) corresponds to the maximum energy deposited by the $511 \mathrm{keV}$ photons. A strong enhancement for the cases when the TOT is below $10 \mathrm{~ns}$ is not only composed from the contribution of prompt and annihilation photons but also due to the interaction of scattered photons. For the PALS studies, in order to estimate the lifetime of the Ps atoms, strong cuts can be applied in a narrow window around the Compton edges of $17.5 \mathrm{~ns}$ (for the selection of annihilation candidate) and a TOT range between 25-35 ns (for the candidates of prompt photon). The time difference in the registration of prompt photon and the average hit time of annihilation photons gives access to estimate the lifetime of Ps atoms.

\section{Summary}

J-PET detector is made of plastic scintillators in which photons interact via the Compton scattering. In the present work, the analysis procedure explaining the selection criteria of the photons of different energies and origins while using the ${ }^{22} \mathrm{Na}$ source surrounded by the XAD4 material is discussed. The precise identification of prompt and annihilation photons is very crucial to perform the tests based on the lifetime of the Ps atoms.

\section{Acknowledgments}

We acknowledge the support by the Foundation for Polish Science through the MPD and TEAM POIR.04.04.00-00-4204/17, the National Science Centre through the grant No. 2016/21/B/ST2/01222, $2017 / 25 / \mathrm{N} / \mathrm{NZ1} / 00861$, the Ministry for Science and Higher Education through grants no. 6673/IA/SP/2016, 7150/E-338/SPUB/2017/1, 7150/E-338/M/2017, 7150/ E-338/M/2018, and 7150/E-338/M/2019.

\section{References}

[1] P. Moskal, Sz. Niedzwiecki, T. Bednarski, et al., Nucl. Instrum. Methods Phys. Res. A 764, 317 (2014).

[2] P. Moskal, N. Zon, T. Bednarski, et al., Nucl. Instrum. Methods Phys. Res. A 775, 54 (2015).

[3] P. Moskal, O. Rundel, D. Alfs, et al., Phys. Med. Biol. 61, 2025 (2016).

[4] Sz. Niedzwiecki, P. Bialas, C. Curceanu, et al., Acta Phys. Pol. B 48, 1567 (2017).

[5] P. Kowalski, W. Wislicki, R.Y. Shopa, et al., Phys. Med. Biol. 63, 165008 (2018).

[6] D.R. Nygren, "Converting vice to virtue: can timewalk be used as a measure of deposited charge in silicon detectors?", Internal LBL note, 1991.

[7] I. Kipnis, T. Collins, J. DeWitt, et al., IEEE Trans. Nucl. Sci. 44, 289 (1997).

[8] T. Fujiwara, H. Takahashi, K. Shimazoe, B. Shi, IEEE Trans. Nucl. Sci. 57, (2010).

[9] P.D. Olcott, C.S. Levin, in: IEEE Nucl. Sci. Symp. Conf. Record, Dresden 2008 p. 4530.

[10] H. Kim, C.-M. Kao, Q. Xie, C.-T. Chen, L. Zhou, F. Tang, H. Frisch, W.W. Moses, W.S. Choong, Nucl. Instrum. Methods Phys. Res. A 602, 618 (2009).

[11] K. Dulski, B. Zgardzinska, P. Bialas, et al., Acta Phys. Pol. A 132, 1637 (2017).

[12] K. Dulski, C. Curceanu, E. Czerwinski, et al., Hyperfine Interact. 239, 40 (2018). 
[13] P. Moskal, D. Kisielewska, C. Curceanu, et al., Phys. Med. Biol. 64, 055017 (2019).

[14] P. Moskal, B. Jasinska, E.L. Stepien, S.D. Bass, Nature Rev. Phys. 1, 527 (2019).

[15] M. Palka, P. Strzempek, G. Korcyl, et al., JINST 12, P08001 (2017).

[16] G. Korcyl, P. Bialas, C. Curceanu, et al., IEEE Trans. Med. Imag. 37, 2526 (2018).
[17] W. Krzemien, A. Gajos, A. Gruntowski, et al., Acta Phys. Pol. A 127, 1491 (2015).

[18] W. Krzemien, D. Alfs, P. Bialas, et al., Acta Phys. Pol. B 47, 561 (2016).

[19] E. Czerwinski, K. Dulski, P. Bialas, et al., Acta Phys. Pol. B 48, 1961 (2017). 